\title{
DEVELOPMENT OF A MYOELECTRIC CONTROLLER BASED ON KNEE ANGLE ESTIMATION
}

\author{
Alberto López Delis, João Luiz Azevedo de Carvalho, Adson Ferreira da Rocha \\ Francisco Assis de Oliveira Nascimento and Geovany Araújo Borges \\ Department of Electrical Engineering, Universidade de Brasilia, Brasilia, DF, Brazil \\ lopez_delis@yahoo.com,joaoluiz@gmail.com,adson@unb.br,assis@unb.br,gaborges@ene.unb.br
}

\begin{abstract}
Keywords: Electromyographic signal, Prosthesis control, Microcontrolled bioinstrumentation, Feature extraction, Dimensionality reduction, Neural network.

Abstract: $\quad$ This paper presents the development of a bioinstrumentation system for the acquisition and pre-processing of surface electromyographic (SEMG) signals, as well as the proposal of a myoelectric controller for leg prostheses, using algorithms for feature extraction and classification of myoelectric patterns. The implemented microcontrolled bioinstrumentation system is capable of recording up to four SEMG channels, and one electrogoniometer channel. The proposed neural myoelectric controller is capable of predicting the intended knee joint angle from the measured SEMG singals. The controller is designed in three stages: feature extraction, using auto-regressive model and amplitude histogram; feature projection, using self organizing maps; and pattern classification, using a Levenberg-Marquadt neural network. The use of SEMG signals and additional mechanical information such as that provided by the electrogoniometer may improve precision in the control of leg prostheses. Preliminary results are presented.
\end{abstract}

\section{INTRODUCTION}

The use of microprocessors in myoelectric control has grown notably, benefitting from the functionality and low cost of these devices. Microprocessors provide the ability to employ advanced signal processing and artificial intelligence (AI) methods as part of a control system, while easily conforming to control options, and adjusting to the input characteristics. They also provide the ability to implement pattern-recognition-based control schemes, which increases the variety of control functions, and improves robustness.

Surface electromyographic (SEMG) signals provide a non-invasive tool for investigating the properties of skeletal muscles (Sommerich et al, 2000). The bandwidth of the recorded potentials are relatively narrow $(50-500 \mathrm{~Hz})$, and their amplitude is low $(50 \mu \mathrm{V}-5 \mathrm{mV})$ (De Luca, 2006). These signals have been used not only for monitoring muscle behavior during rehabilitation programs (MonseniBendpei et al, 2000), but also for the mechanical control of prostheses. In this context, it is important to be able to correctly predict which movement is intended by the user. The SEMG signal is very convenient for such application, because it is non- invasive, simple to use, and intrinsically related to the user's intention. However, there are other useful variables, especially those related to proprioception, for example: the angle of a joint, the position of the limb, and the force being exerted.

This project is supported under the development of an active leg prosthesis prototype (Figure 1). The prosthesis has three degrees of freedom: one for the knee (sagittal plane), and two movements for the foot (sagittal and frontal plane). The three degrees of freedom are associated to the angles $\theta_{1}, \theta_{2}$ and $\theta_{3}$, controlled by $\mathrm{DC}$ reduction motors.

The prototype will be fixed to the patient's upper leg through a fixing capsule, where the SEMG sensors will be located. The prosthesis will receive control commands through digital signal processing, feature extraction, and pattern classification.

Specifically, for the development of an active leg prosthesis that also possesses ankle and foot axes, it is necessary to use other sources of information besides SEMG (Ferreira et al, 2005). Thus, the use of myoelectric signals combined with other variables related to proprioception may improve the reliability in closed-loop control systems. In addition, the bioinstrumentation system should be as immune to noise and interference as possible. This can be 
achieved by proper board and shielding design, as well as the use of filters whenever they are necessary.

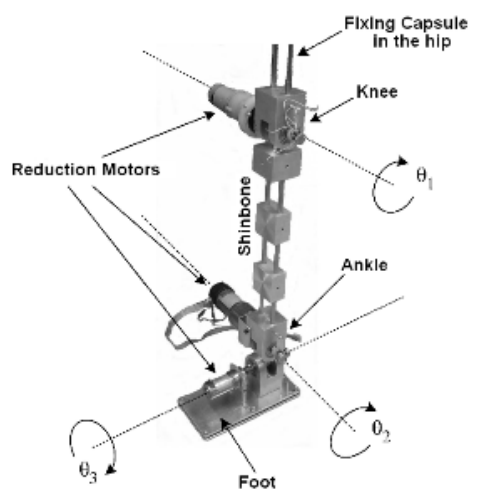

Figure 1: Mechanical structure of the prosthesis prototype.

Figure 2 presents the typical main components of a general myoelectric controller based on pattern recognition. The SEMG signals are acquired by surface electrodes placed on the skin over muscle(s) of the user. The signals originating from the electrodes are pre-amplified to differentiate the small signals of interest, and then are amplified, filtered and digitized. Finally, the information is transferred to a myoelectric controller (Asghari and $\mathrm{Hu}, 2007)$.

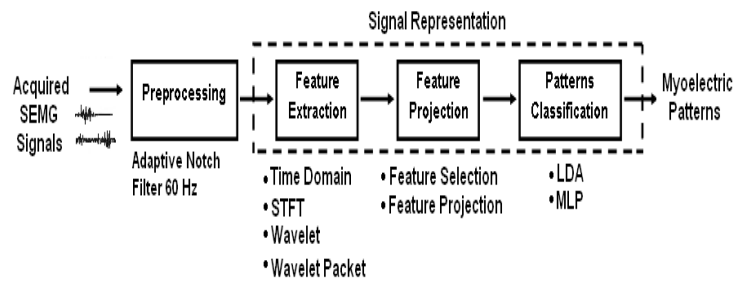

Figure 2: Typical main components of a general myoelectric controller based on pattern recognition.

In the design and implementation of a myoelectric controller, the system's precision is essential for a realistic accomplishment of the user's intention. The precision is an important factor on the development of multi-sensory controllers, and can be improved by extracting more information from the muscle's state, and using a classifier that is capable of improving this information. The controller should be capable of learning the muscular activation patterns that are used in natural form for typical movements. It also needs robustness against the condition variations during the operation. The response time cannot create delays that are noticeable to the user.

This article presents a micro-controlled bioinstrumentation prototype system as part of the development of an active leg prosthesis structure that allows the acquisition and processing of electromyographic signals and other data related to the articulate movement, specifically the angle of the knee. The information obtained is processed in order to obtain appropriate myoelectric patterns for prosthesis control. Preliminary results on the design of algorithms for the estimation of the knee angle, based on patterns recognitions techniques, are presented.

\section{METHODS}

The front end stage of the designed bioinstrumentation system acquires up to four SEMG channels. The SEMG are measured on a pair of agonist and antagonist muscles of the leg (Fig. 3). An electrogoniometer is used to measure the flexion and extension angles of the knee joint (Fig. 3c).

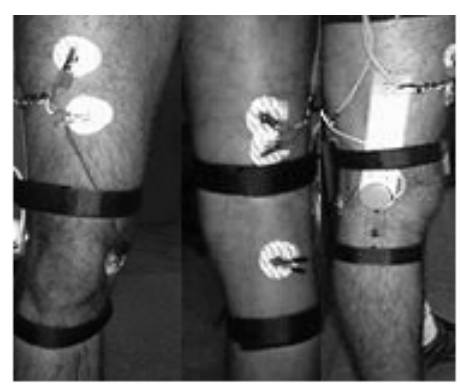
(a)
(b)
(c)

Figure 3: Experimental setup. Surface electrodes are placed over a pair of agonist and antagonist muscle groups of the leg: (a) vastus intermedius, (b) semitendinosus. An electrogoniometer is used to measure the flexion and extension angles of the knee joint (c).

Differential amplifiers, used in the bipolar configuration, significantly reduce the common mode interference signals $(\mathrm{CMRR}>110 \mathrm{~dB})$. A band-pass filter between $10 \mathrm{~Hz}$ and $500 \mathrm{~Hz}$ frequency range is used. It is composed by a lowpass filter and high-pass filter with a programmable gain stage from digital potentiometers, controlled by the microcontroller. These elements allow the setting of the SEMG amplitude levels based on the measurements from the patient. To minimize power consumption and increase noise immunity, operational amplifiers with JFET inputs were used. To obtain adequate myoelectric amplitude, an overall gain of up to 20000 can be programmed at the front end (De Luca, 2006).

A second block, micro-controlled and optically isolated from the front end (Figure 4), centralizes all 
the functions associated with the analog/digital conversion process, implementing the digital gain control for the front end amplifiers and synchronized sampling of SEMG signals. The microcontrollers belong to the ARM SAM7S64 ATMEL family of high performance processors, based on 32-bit RISC architecture with an integrated group of peripherals that minimize the number of external components.

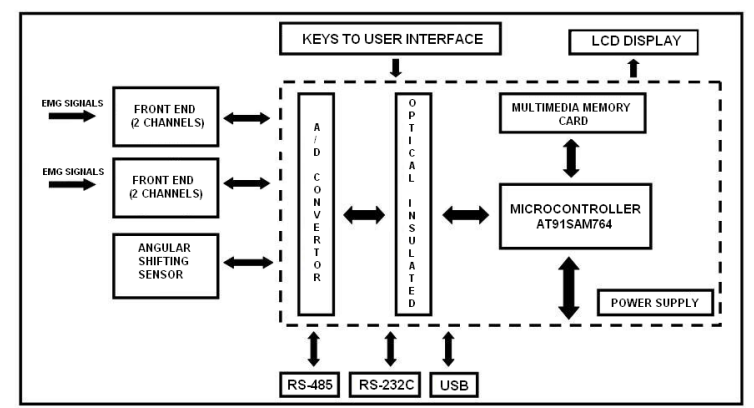

Figure 4: Block diagram of the bioinstrumentation system.

A 13-bit A/D converter with Serial Peripheral Interface (SPI) is used for signal sampling, and allows discriminating small amplitude levels. The electrogoniometer channel is coupled to the system, and generates an electric signal corresponding to the angular position ranging from 30 to 240 degrees. The sampling frequency of each channel is 1744.25 Hz. Figure 5 presents example data acquired during an experimental measurement.

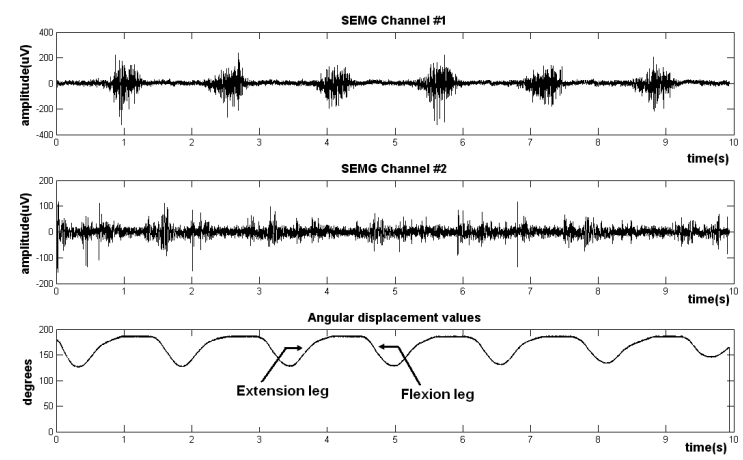

Figure 5: Recorded SEMG signals (rectus femoris and opposite muscles) and angle of the knee joint during a 10 -second experiment.

The microcontroller is linked through RS-485 protocol to the central processor of the prosthesis, which is responsible for coordinating the tasks in the control process. Besides the RS485 protocol, which provides the interaction of the block with the central processor, RS-232C and USB interfaces are available for the communication with a $\mathrm{PC}$ when the system is configured in stand alone mode (Figure 6). In this mode, the system allows the visualization of the state of the experiments during their realization using a LCD display. The instrumentation system is designed using low power consumption components, which increases the system's portability.

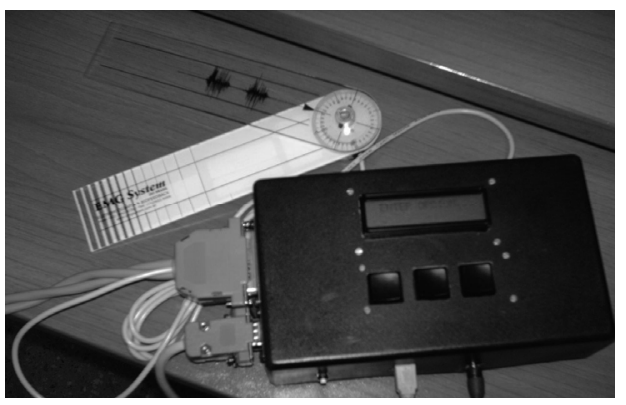

Figure 6: Bioinstrumentation module (with accessories) configured in stand alone mode.

\subsection{Adaptive Filter Implementation}

The power line interference usually has its first harmonics $(60 \mathrm{~Hz}, 120 \mathrm{~Hz}, 180 \mathrm{~Hz}$, and $240 \mathrm{~Hz})$ in a portion of the spectrum with major SEMG energy concentration. The use of an analog notch filter may distort the signal; therefore it should only be used when really necessary. Generally, the best option is to use an adaptive notch filter. An embedded subroutine in the ARM-SAM7S64's core implements an adaptive notch filter in real time. This filter maintains a running estimate of the $60 \mathrm{~Hz}$ interference, and the current noise at time $t$ can be estimated from the previous two noise estimates (Hamilton, 1996), as shown in equations (1) and (2),

$$
e(t)=N e(t-n T)-e(t-2 n T)
$$

where $T$ is the sample period and $N=2 \cos (2 \pi .60 . T)$. In the filter, the output is generated by subtracting the estimated noise, $e(t)$, from the input signal, $x(t)$. The expression presented in equation (2) is used to implement the filter.

$$
f(t)=[x(t)-e(t)]-[x(t-n T)-e(t-n T)]
$$

If $f(t)>0$, then the estimate was too low, so we adjust the estimate upward by incrementing $d$ :

$$
e(n T+T)=e(n T+T)+d
$$

If $\mathrm{f}(\mathrm{t})<0$, the estimate was too high, so the estimate is decremented:

$$
e(n T+T)=e(n T+T)-d
$$


As $d$ increases, the filter adapts more rapidly, and exhibits a broad bandwidth. Similarly, as $d$ decreases, the filter adapts more slowly, and has a narrower bandwidth. The selection of the $d$ factor is empiric, based on test realizations, and its value is small compared to the dynamic range of the $A / D$ converter (Hamilton, 1996). Figure 7 shows the adaptive filtering of a SEMG signal measured on the rectus femoris muscle.
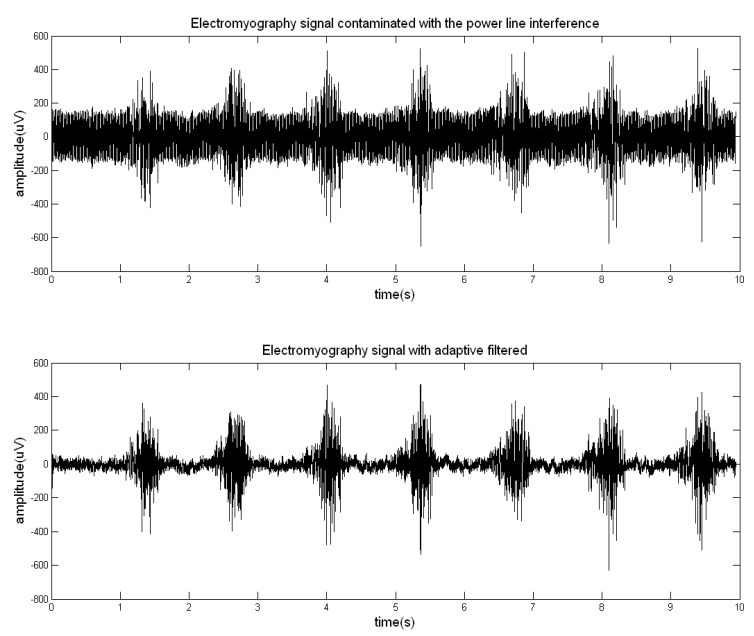

Figure 7: Adaptive filtering performed on a SEMG signal contaminated with power-line interference.

\subsection{Myoelectric Controller}

Presenting the myoelectric signal directly to a classifier is impractical, because of the dimensionality and the random characteristics of the signal. It's necessary that the signal is represented as a vector with reduced dimensionality, i.e., a feature vector. The myoelectric controller algorithm proposes the use of three stages for feature extraction and pattern classification. The first stage consists in the mixture of feature vectors from time domain and spectral analysis. A second stage will perform the reduction of the feature space, allowing the increase in the number of SEMG input sensors without affecting the performance of the control process. The last stage has the goal of estimating the knee angle.

\subsubsection{Feature Vector Extraction}

Given the stochastic nature of the myoelectric signal, it can be considered as a time series, and modeled as a linear combination of their past and present values. The autoregressive model is a convenient structure for model identification, especially when the computations of velocity and response time are important, as in the recognition and classification of myoelectric patterns. The autoregressive coefficients provide information about the muscular contraction. The estimate of the coefficients is performed using a recursive least squares (RLS) technique, with a forgetting factor. This method gives more weight to the most recent samples at the moment of the iteration cycle. The parameters are calculated recursively (Ljung, 1987), as presented in equations (5), (6) and (7):

$$
\begin{gathered}
\hat{\eta_{k}}=\hat{\eta_{k-1}}+L_{k}\left[y_{k}-\varphi_{k}^{T} \eta_{k-1}^{\wedge}\right] \\
P_{k}=\left[P_{k}-\frac{P_{k-1} \varphi_{k} \varphi_{k}^{T} P_{k-1}}{\lambda_{k} \varphi_{k}^{T} P_{k-1} \varphi_{k}}\right] \frac{1}{\lambda_{k}} \\
L_{k}=\frac{P_{k-1} \varphi_{k}}{\lambda_{k} \varphi_{k}^{T} P_{k} \varphi_{k}}
\end{gathered}
$$

where $\hat{\eta}_{k}$ are the vector coefficients that are estimated at discrete time $k ; \varphi_{k}$ are the regressive vectors, $P_{k}$ is the inverse correlation matrix and $L_{k}$ is the gain vector of the filter. The forgetting factor $\lambda_{k}$ controls the system response time. The coefficient estimated at instant $k$ can be interpreted as a characteristic of the SEMG signal within the time interval specified by the forgetting factor, and it is a way of determining the angular displacement that the patient wants to impose to the prosthesis (Ferreira et al, 2005). The coefficients form a feature vector for the pattern classification processes.

Recent research (Hargrove et al, 2008) has demonstrated that a functional and efficient configuration consists of a mixture of feature vectors on the time domain with autoregressive coefficients. This configuration provides good classification precision, and is computationally efficient, which facilitates its implementation in embedded systems. It is also more robust to the displacement of the surface electrodes (Hargrove et al, 2008).

This work uses a mixture of the autoregressive vector with the EMG Histogram method. The EMG Histogram is an extension of the Zero Crossing and the Willison amplitude (Zardoshti-Kermai et al, 1995). Myoelectric signals reach relatively higher levels during the contraction process, compared to the base line amplitudes. Thus, vectors obtained from the histogram provide a measure of the frequency in which the signal reaches each level of amplitude, associated with different histogram bins. For the implementation of the histogram, a 
symmetrical interval with respect to base line over the SEMG register is established and the same is subdivided into 9 bins. These bins represent intervals of amplitude in which the SEMG signal is grouped.

The resultant feature vectors (autoregressive and histogram) are concatenated, and then used as the input vector of the feature projection stage.

\subsubsection{Feature Projection}

A feature projection stage is used to reduce the dimension of the feature space of the input vectors, before pattern classification process, using supervised neural networks. This reduction is performed using an unsupervised Kohonen self-organizing map (SOM) neural network. The groups of vector coefficients obtained from each SEMG channel using the RLS and histogram methods are transformed into two-dimensional vectors. With the reduction in input dimension, the SOM is able to reduce noise and absorb the large variations that appear in the original features. In addition, the SOM can shorten the training time of the supervised neural network. The unsupervised SOM can find the winning neuron on a 2-D map to represent the original pattern. To find the output neuron (winning node), the following steps are used, according to the learning rule of the Kohonen Feature map (Haykin, 1999).

Step 1: Choose random values for the initial weight vectors $W_{j}(0)$.

Step 2: Find the winning neuron $y_{c}$ at time step $t$ (Similarity Matching), by using the minimumdistance Euclidean criterion:

$$
y_{c}=\operatorname{argmin}\left\|x(t)-W_{j}(t)\right\|, j=1,2, \ldots ., t
$$

Step 3: Update the synaptic weight vectors of all neurons by using the following update rule:

$$
W_{j}(t+1)=W_{j}(t)+\eta(t) h_{j, y_{c}}(t)\left[x(t)-W_{j}(t)\right]
$$

where $\eta(t)$ is the learning rate, and $h_{j, y_{c}}(t)$ is the neighbors function centred around the winner. $\eta(t)$ and $h_{j, y_{c}}(t)$ are varied dynamically during the learning stage, in order to obtain optimal results.

Step 4: Go back to step 2 until no changes in the feature map are observed.

The inputs of the Kohonen's SOM are features from each channel, and the output is the 2-D coordinate (on the $x$ and $y$ axes) on the 2-D topological net. A 2-D coordinate is a condensed feature for each channel (Figure 8).

\subsubsection{Myoelectric Classification}

Multi-layer neural networks have been successfully applied to many difficult and nonlinear problems in diverse domains and there is considerable research on methods to accelerate the convergence time of the multi-layer feedforward neural network algorithm (Battiti, 1992 and Charalambous, 1992). The method used in this paper is the LevenbergMarquadt (LM) algorithm (Hagan and Menhaj, 1994), which consists in the use of the nonlinear least squares algorithm to the batch training of multi-layer perceptrons. The LM algorithm can be considered a modification of the Gauss-Newton method. The key step in the LM algorithm is the computation of the Jacobian matrix. The LM algorithm is very efficient when training networks which have up to a few hundred weights. Although the computational requirements of the LM algorithm become much higher after each iteration, this is fully compensated by its higher efficiency. This is especially true when high precision is required (Hagan and Menhaj, 1994). Figure 8 presents the complete block diagram of the myoelectric controller.

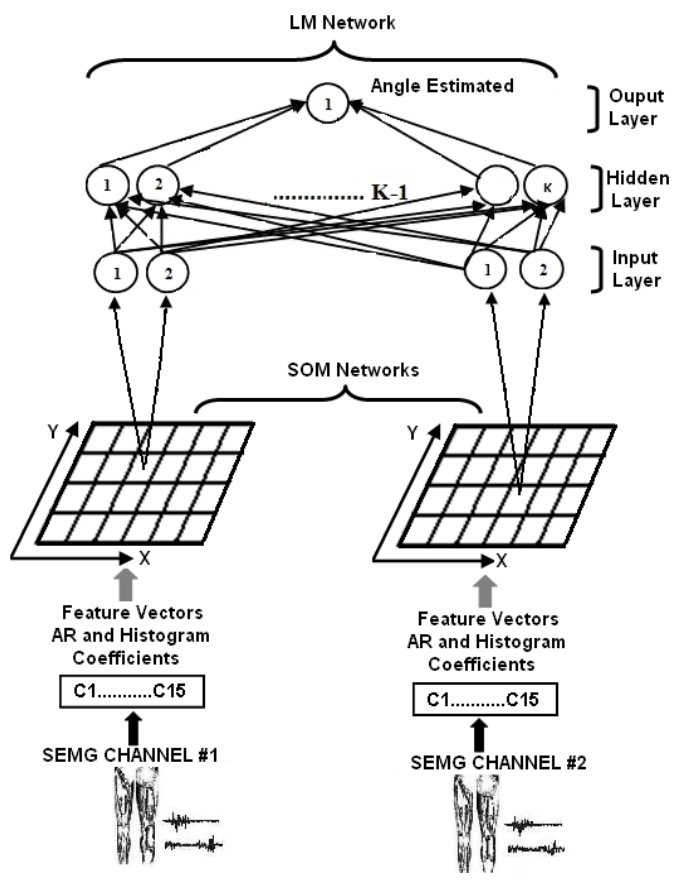

Figure 8: Block diagram of the proposed myoelectric controller algorithm. 


\section{RESULTS}

As a prototype implementation, the training and validation processes were performed in off-line mode, and the algorithms described above were implemented in Matlab. At a later stage, the full validation of the controller will be the executed from an embedded system running on a Linux platform.

For this demonstration, SEMG measurements were captured from a healthy subject using $10 \mathrm{~mm}$ $\mathrm{Ag} / \mathrm{AgCL}$ surface electrodes placed on a pair of antagonistic muscles, associated with the flexing and extension movements of the knee (Figure 3). The electrodes were arranged in bipolar configuration, and gel was used to reduce the resistance between electrodes and skin. The distances between the centers of the electrodes was 3-5 $\mathrm{mm}$, and the reference electrode was placed over the lateral condyle bone. A total of 10 measurements were performed, divided in two groups of signals training and validation - acquired during walks with different speeds, with duration of 10 seconds.

For training purposes, it is essential to know information about the input and output, comparing the dimensional vectors obtained from the SOM network to the displacement angle measured by the electrogoniometer sensor. Figure 9 shows the estimated angle compared to the measured angle from the electrogoniometer. Although the estimated angle follows the measurement satisfactorily, the output of the LM network presents the impulsive noise (9a), which is canceled using a moving average recursive filter with a 50-sample window (9b). This filter keeps the changes levels or slopes that are present in the angle estimated and present a
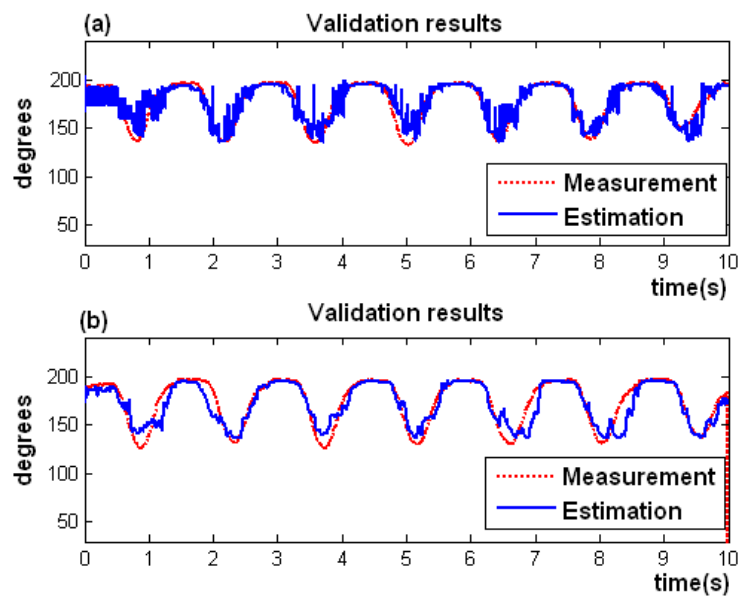

Figure 9: Comparison of the estimated knee angle (blue) to the measured angle from the electrogoniometer (red): (a) before filtering; (b) after filtering. delay of $(M-1) / 2$ samples, where $M$ is the number of samples in the average (Smith, 1999). The results obtained with 50 samples of average were satisfactory, decreasing the variance and conserving the changes levels (Figure 9b).

A preliminary comparison was performed between the proposed algorithm and the methods proposed by Ferreira et al. (2005). The proposed algorithm it is an alternative to the latter approach, which consists in using the AR model for feature extraction, and a LM multi-layer perceptron neural network for pattern classification. The evaluation was based on the classification error, which was calculated using the following equation:

$$
\text { error }=\sqrt{\frac{\sum_{i=1}^{N}|x(i)-\hat{x(i)}|^{2}}{\sum_{i=1}^{N}|x(i)|^{2}}} \times 100(\%)
$$

where $x(i)$ and $x(i)$ represent the angular values from the electrogoniometer sensor and the angle estimated vectors respectively, $N$ is the dimension of the vectors. Table 1 presents the averages error rate of classification measured in the group of validation signals.

The results in Table 1 show that the proposed algorithm achieved better classification then the method proposed by Ferreira et al. (2005). This means that the proposed method is more accurate in estimating the knee joint angle from the myoelectric signals.

Table 1: Comparison based the average rate classification.

\begin{tabular}{|c|c|}
\hline Ferreira et al. $(2005)$ & $8.02 \% \pm 4.2$ \\
\hline Proposal Algorithm & $5.86 \% \pm 1.6$ \\
\hline
\end{tabular}

\section{CONCLUSIONS}

This paper presents the current state of development of a bioinstrumentation system for active control of leg prostheses. Features of the system and of the signal processing algorithm used in the myoelectric controller were presented. The system allows the acquisition of SEMG signals with a maximum amount of signal information and a minimum amount of contamination from electrical noise. The results show that the system has great potential for future developments in leg prostheses. Another feature of the system is that it works not only as a part of the prosthesis control, but also in stand alone mode. Preliminary analysis showed that the 
computational complexity of the proposed algorithm increases for each iteration during execution of the LM network. Future work will aim optimize the code for its execution in real time.

\section{ACKNOWLEDGEMENTS}

This work was partially supported by CAPES and CNPq.

\section{REFERENCES}

Asghari, M. O. and Hu, H. 2007. Myoelectric Control System - A survey, Biomedical Signal Processing and Control, 2 275-294.

Battiti, R. 1992. First and second order methods for learning: Between steepest decent and Newton's method, Neural Computation, 4 141-166.

Charalambous, C. 1992. Conjugate gradient algorithm for efficient training of artificial neural networks, Circuit, Devices and Systems, IEE Proceedings, 301-310.

De Luca, 2006. Encyclopedia of Medical Devices and Instrumentation, John G. Webster Ed., 98-109.

Ferreira, R. U., da Rocha, A. F., Casão, Jr C. A., Borges, G. A., Nascimento, F. A. O., Veneziano, W. H. , 2005. Reconhecimento de Padrões de Sinais de EMG para Controle de Prótese de Perna, Proc. XI Congresso Brasileiro de Biomecânica, Brasil.

Hagan, M. T. and Menhaj, M. B. 1994. Training feedforward networks with the Marquadth Algorithm, IEEE Trans. Neural Networks 5 989-993.

Hamilton, P. S. 1996. A Comparison of Adaptive and Nonadaptive Filters for Reduction of Power Line Interference in the ECG, IEEE Trans. Biomed. Eng., 43 105-109.

Hargrove, L., Englehart, K. and Hudgins, B. 2008. A training strategy to reduce classification degradation due to electrode displacements in pattern recognition based myoelectric control, Biomedical Signal Processing and Control, 3 175-180.

Haykin, S. 1999. Neural Networks: A Comprehensive Foundation, New Jersey: Prentice Hall.

Ljung, L. 1987. Linear System Identification, PrenticeHall, Inc: Englewood Cliffs.

Monseni-Bendpei, M.A., Watson, M.J. and Richardson, B. 2000. Application of surface electromyography in the assessment of low back pain: a literature review. Phys. Ther. Rev., 2 93-105.

Sommerich, C.M., Joines, S.M., Hermans, V. and Moon, S.D. 2000. Use of surface electromyography to estimate neck muscle activity, J. Electromyography Kinesiology, 6 377-398.

Smith, S. W. 1999. The Scientist and Engineer's Guide to Digital Signal processing, 2(ed.), San Diego: California Technical Publishing.
Zardoshti-Kermani, M., Wheeler, B. C., Badie, K. and Hashemi, R. M. 1995. EMG Feature Evaluation for Movement Control of Upper Extremity Prostheses, IEEE Trans. on Rehabilitation Eng., vol 3 324-333. 